\title{
用于合成气制烯烃的疏水性FeMn@Si催化剂
}

\author{
徐艳飞, 定明月* \\ 武汉大学动力与机械学院, 武汉 430072 \\ *联系人, E-mail: dingmy@whu.edu.cn
}

\section{A hydrophobic FeMn@Si catalyst for syngas to olefins}

\author{
Yanfei Xu \& Mingyue Ding* \\ School of Power and Mechanical Engineering, Wuhan University, Wuhan 430072, China \\ * Corresponding author, E-mail: dingmy@whu.edu.cn \\ doi: 10.1360/TB-2021-0230
}

烯烃在能源化工领域是一类非常重要的基础化工原料, 可用其合成橡胶、塑料、纤维、润滑油等大宗产品，在国民 经济中占有十分重要的地位. 近年来, 随着烯烃需求量的日 益增加和石油资源的逐渐枯竭，开发非石油基生产烯烃的路 线备受关注. 基于我国“富煤、贫油”的能源格局, 发展煤制烯 烃技术逐渐成为了能源化工界的主流. 费-托(F-T)合成反应 可将煤炭资源经合成气 $\left(\mathrm{CO}\right.$ 和 $\left.\mathrm{H}_{2}\right)$ 转化为液体燃料和高碳化 学品, 该技术已主导煤化工领域近百年. 然而该过程中通常 发生水煤气变换反应(WGS), 产生大量的 $\mathrm{CO}_{2}$, 导致后续能耗 大幅增加. 另外, F-T合成是强放热反应, 容易导致 $\mathrm{CH}_{4}$ 的生 成, 降低高碳产品的收率. 在F-T合成过程中, 约 $50 \%$ 的 $\mathrm{CO}$ 转 化成了 $\mathrm{CO}_{2}($ 约 $40 \%)$ 和 $\mathrm{CH}_{4}$ 等 $\mathrm{C} 1$ 副产物, 碳原子利用效率低下, 严重降低了能源效益和经济效益. 如何高效降低F-T合成过 程中 $\mathrm{C} 1$ 副产物的生成, 提高特定烃类产品的选择性一直是国 际能源化工界的巨大挑战之一. 近期, 本课题组 ${ }^{[1]}$ 报道了一种 疏水性FeMn@Si-c催化剂，可以有效抑制合成气制烯烃过程 中 $\mathrm{C} 1$ 副产物的生成, 并揭示了碳化铁活性相与疏水基团的高 效协同对于抑制WGS反应、降低 $\mathrm{CO}_{2}$ 排放量至关重要，该研 究成果发表于Science.

通过Stöber法在氧化铁纳米颗粒表面均匀包覆了一层无 定形氧化硅, 进一步的表面功能化修饰将氧化硅壳层上的亲 水性基团 $(-\mathrm{OH})$ 替换成疏水性基团 $\left(-\mathrm{CH}_{3}\right)$. 有机官能团修饰 使得催化剂由亲水性转变为疏水性(图1(a)), 有效抑制了水分 子在催化剂上的吸附与滞留. 通过分析反应后催化剂的物相 组成, 本研究发现催化剂表面亲水、疏水性质的改变可有效 调控F-T合成反应中含铁物相的转变行为. 新鲜亲水性 $\mathrm{Fe} @ \mathrm{Si}$ 和疏水性 $\mathrm{Fe} @ \mathrm{Si}$-c 催化剂均以 $\mathrm{Fe}_{2} \mathrm{O}_{3}$ 相为主, 在预处理过程中 可进一步转变为 $\mathrm{Fe}_{x} \mathrm{C}$ 相. 在反应过程中水的作用下, 亲水性
$\mathrm{Fe} @ \mathrm{Si}$ 催化剂中部分 $\mathrm{Fe}_{x} \mathrm{C}$ 氧化为 $\mathrm{Fe}_{3} \mathrm{O}_{4}$, 最终形成了 $\mathrm{Fe}_{3} \mathrm{O}_{4}$ 和 $\mathrm{Fe}_{x} \mathrm{C}$ 的混合物相(图1(b)), 这与传统 $\mathrm{Fe}$ 基催化剂相一致. 疏水 性 $\mathrm{Fe} @ \mathrm{Si}$-c 催化剂在反应过程中保持 $\mathrm{Fe}_{x} \mathrm{C}$ 相不变、没有 $\mathrm{Fe}_{3} \mathrm{O}_{4}$ 相生成(图1(c)), 说明疏水性氧化硅壳层可以有效抑制水分子 在催化剂上的吸附与滞留, 进而抑制水对核层 $\mathrm{Fe}_{x} \mathrm{C}$ 的氧化. $\mathrm{Fe}_{x} \mathrm{C}$ 被广泛认为是F-T合成反应的活性相, 进一步说明疏水 性 $\mathrm{Fe} @ \mathrm{Si}$-c 催化剂可保持 $\mathrm{Fe}_{x} \mathrm{C}$ 活性相在反应过程中处于良好 的稳定状态.

进一步, 我们研究了催化剂表面亲水、疏水性质的调变 对F-T合成反应过程中 $\mathrm{CO}_{2}$ 选择性的影响. 疏水性氧化硅壳层 可以抑制反应过程中水分子在催化剂上的吸附与滞留，降低 核层铁物种附近的局部水分压, 进而抑制了水与 $\mathrm{CO}$ 进一步通 过WGS反应生成 $\mathrm{CO}_{2}$. 因此, 在 $\mathrm{CO}_{2}$ 选择性- $\mathrm{CO}$ 转化率关系图 中, 疏水性 $\mathrm{Fe} @ \mathrm{Si}-\mathrm{c}$ 催化剂上的 $\mathrm{CO}_{2}$ 的选择性显著低于亲水性 $\mathrm{Fe} @ \mathrm{Si}$ 催化剂(图1(d)). 在此基础上, 通过Mn助剂进一步调节 F-T合成反应的产物分布(图1(e)). 研究结果表明, 适量Mn助 剂的添加并不破坏催化剂的织构性质. Mn 金属向Fe活性金属 表界面的电子转移有利于 $\mathrm{CO}$ 的吸附、抑制 $\mathrm{H}_{2}$ 的吸附, 显著 提高了烯烃产物的选择性, 并抑制了副产物 $\mathrm{CH}_{4}$ 的生成, 同时 保持较低的 $\mathrm{CO}_{2}$ 选择性. 然而, 添加过量 $\mathrm{Mn}$ 助剂阻碍了疏水 壳与内部铁物种之间的有效接触, 导致 $\mathrm{Fe}_{x} \mathrm{C}$ 被部分氧化为 $\mathrm{Fe}_{3} \mathrm{O}_{4} . \mathrm{Fe}_{3} \mathrm{O}_{4}$ 被认为是含铁物相中对WGS反应活性最高的物 相, 在F-T合成反应过程中, $\mathrm{Fe}_{x} \mathrm{C}$ 向 $\mathrm{Fe}_{3} \mathrm{O}_{4}$ 的转变则明显促使 了WGS反应的发生, 从而导致 $\mathrm{CO}_{2}$ 选择性的显著增加. 与其 他合成气制烯烃的催化体系 ${ }^{[2 \sim 6]}$ 相比(图1(f)), FeMn@Si-c催 化剂实现了在较高转化率水平下对 $\mathrm{C} 1$ 副产物的有效抑制.

核层碳化铁活性相与壳层疏水基团的高效协同, 可以拓 展出一系列新型的复合催化剂, 通过抑制高耗能的水煤气变 



图 1 (网络版彩色)(a) 催化剂的水滴接触角测试. 反应后 $\mathrm{Fe} @ \mathrm{Si}(\mathrm{b})$ 和Fe@Si-c(c)的穆斯堡尔谱. (d) $\mathrm{CO}_{2}$ 选择性-CO转化率关系图. (e) Mn含量对 催化剂产物分布的影响. (f) 与其他催化剂的反应性能对比

Figure 1 (Color online) (a) Water-droplet contact angle tests. Mössbauer spectra of the spent Fe@Si (b) and Fe@Si-c (c). (d) $\mathrm{CO}_{2}$ selectivity-CO conversion curve. (e) Effect of $\mathrm{Mn}$ content on the products distribution of catalysts. (f) Comparison of products distribution with other catalysts

换反应，大幅度降低合成气转化过程中的二氧化碳排放，显 著提高碳原子利用效率，有望实现用合成气更高效、更经济 地制取烯烃、汽油、芳烃、航油等各种高附加值化学品. 荷 兰格罗宁根大学Jingxiu Xie教授受邀在Science上撰写了题为
“Designing the right protection”的亮点评论文章, 认为“这种疏 水性界面催化剂的构建非常巧妙, 为其他类型需要避免水作 用的相关反应提供了一个新思路”, ${ }^{[7]}$. 该研究成果对于实现 “碳中和”目标提供了新的解决方案.

\section{推葆阅读文献}

1 Xu Y, Li X, Gao J, et al. A hydrophobic FeMn@Si catalyst increases olefins from syngas by suppressing C1 by-products. Science, 2021, 371: 610613

2 Jiao F, Li J, Pan X, et al. Selective conversion of syngas to light olefins. Science, 2016, 351: 1065-1068

3 Torres Galvis H M, Bitter J H, Khare C B, et al. Supported iron nanoparticles as catalysts for sustainable production of lower olefins. Science, 2012, 335: $835-838$

4 Zhong L, Yu F, An Y, et al. Cobalt carbide nanoprisms for direct production of lower olefins from syngas. Nature, 2016, 538: 84-87

5 Xie J, Paalanen P P, van Deelen T W, et al. Promoted cobalt metal catalysts suitable for the production of lower olefins from natural gas. Nat Commun, 2019, 10: 167

6 Wang P, Chen W, Chiang F K, et al. Synthesis of stable and low- $\mathrm{CO}_{2}$ selective $\varepsilon$-iron carbide Fischer-Tropsch catalysts. Sci Adv, 2018, 4: eaau2947

7 Xie J. Designing the right protection. Science, 2021, 371: 577 